Rev SINAPSIS, Vol. 6, Nº 1, Junio 2015

ISSN $1390-7832$

\title{
Bioética y calidad de atención en adolescentes atendidas en ginecología de hospitales públicos
}

\section{Bioética y atención a adolescentes en hospitales}

\author{
Mabel Sánchez Msc Ge $\mathrm{G}^{(1)}$ \\ Milton Espinoza Lucas $\mathrm{Msc} \mathrm{IcE}^{(2)}$ \\ Miladis Placencia Msc Um ${ }^{(3)}$ \\ (1)Universidad Laica Eloy Alfaro de Manabí \\ (2) Universidad Laica Eloy Alfaro de Manabí \\ ${ }^{(3)}$ Universidad Estatal del Sur de Manabí.
}

Contacto: dramabelsan24@hotmail.com

Receptado: 10/02/2015 Aceptado: 11/04/2015

\section{Resumen}

Los grupos vulnerables del país son: adultos mayores, embarazadas, discapacitados y otros no adolescentes, razonable por su grado de riesgo, una de las etapas biológicas trascendental es la adolescencia, la fisiología cambia y las definiciones de personalidad se encuentran en proceso .Entonces constituye una etapa vulnerable si no existen condiciones adecuadas de orientación sexual y provisión de condiciones que permitan reconocer riesgos y aplicar estrategias destinadas a disminuir detrimentos en su calidad de vida. Los niveles de atención del sistema de salud ecuatoriano ameritan un personal de salud laborando en instituciones aplicando principios bioéticos en la atención a usuarios, el estudio plantea como objetivo determinar si el personal de enfermería aplica principios bioéticos en la atención a adolescentes atendidas del área ginecológica del hospital básico Jipijapa, se realizó una encuesta a adolescentes y entrevista al personal de salud, se evaluó el nivel de satisfacción de servicios, se identificó los principales dilemas bioéticos que las enfermeras enfrentan en sus actividades y se estableció la no existencia protocolos en caso de suscitarse dilemas bioéticos. Los resultados mostraron que el personal de enfermería conoce misión, visión de los servicios que presta, existen buenas relaciones interpersonales, sin embargo no manejan protocolos al presentarse problemas bioéticos, existe respeto al paciente y familiares .Las adolescentes consideran que las enfermeras no brindan trato 
Rev SINAPSIS, Vol. 6, Nº 1, Junio 2015

individualizado, ni humanístico en algunos casos a pesar satisfacer sus necesidades inmediatas en gran medida y consideran que deben tener capacitaciones para mejorar el trato a pacientes.

Palabras clave: cuidados a adolescentes, principios bioéticos, protocolos de atención

\title{
Bioethics and quality of care in gynecology adolescents treated in public hospitals
}

\begin{abstract}
Vulnerable groups in the country are: the elderly, pregnant women, the disabled and others not teenagers, reasonable by their degree of risk, one of the crucial biological stages of adolescence, physiology changes and the definitions of personality are in then process is a vulnerable stage if there are no adequate conditions of sexual orientation and providing conditions that allow recognize risks and implement strategies to reduce detriment to their quality of life. The levels of health care system merit Ecuadorian health personnel working in institutions applying bioethical principles in the customer first, the study therefore seeks to determine whether nurses bioethical principles applied in the care of adolescents met the gynecological area Jipijapa basic hospital, a survey was conducted to teenagers and interview health personnel, the level of service satisfaction was evaluated, the principal bioethical dilemmas identified that nurses face in their activities and nonexistence was established protocols should arise bioethical dilemmas. The results showed that the nurses called mission, vision of the services provided, there are good relationships, but no protocols to handle bioethical problems occur, there is respect for patients and relatives .The teens believe that nurses do not provide individualized treatment nor humanistic in some cases even their immediate needs greatly and they believe they should have training to improve the treatment of patients.
\end{abstract}

Key words: Adolescent care, bioethical principles, care protocols

\section{Introducción}

Dentro de la planificación del Ecuador en el aspecto de salud está reducir el porcentaje de embarazos en adolescentes a nivel nacional .Cabe señalar que dicho indicador experimentó un incremento que situó a Ecuador como el país con el más alto índice de embarazos adolescentes en América Latina (17\% aproximadamente) el crecimiento en los últimos 10 años bordea el 74\%. (Ecuador, 2012).Esa realidad es similar en países cercanos, desde hace poco tiempo los países de la región se preocupan por dar solución a los problemas sociales, de salud y de derechos de los y las adolescentes y jóvenes. En un análisis de las políticas y la legislación vigentes sobre el tema en nuestra región, realizado en colaboración con la Universidad George Washington, se demostró que la mayoría de los países carecen de políticas públicas de juventud. (Ginecología, 2013) 
Dentro de los involucrados en la atención a adolescentes encontramos además del núcleo familiar y los docentes al personal de salud, entre los que se encuentra el profesional de enfermería, quien debe realizar procesos contando con la opinión de los pacientes en todas las actividades relacionadas a la atención brindada, este personal ha de tratar al paciente con el respeto que merece, lo cual exige establecer un diálogo cuya finalidad sea orientarle y aclarar las dudas que estén relacionadas con los conocimientos de la situación de salud actual de acuerdo al perfil profesional que está brindando el servicio. El paciente adolescente en éste caso tiene derecho a ser escuchado en la toma de decisiones que le puedan afectar, lo cual genera un sin número de implicaciones en el caso de tratamientos preventivos y terapéuticos.

De lo anterior se desprende la importancia que tiene para el profesional de enfermería en el desarrollo de una comprensión empática que significa ponerse en el lugar del otro, hasta sentirse capaz de comprenderle y anticiparse a las respuestas que exige su estado ayudándole de esta manera a actuar de un modo adecuado, ese y el aspecto de la escolaridad es uno de los más afectados en el caso de embarazos adolescentes, además de la insuficiente orientación que las adolescentes reciben en las áreas de salud para asumir o no la maternidad y prevenir futuros embarazos.

Haciendo referencia a esto y a la insuficiente información sobre sexualidad en las adolescentes ,se toma en cuenta también el impacto del entorno familiar, la escolaridad y el embarazo adolescente revisando estudios sobre las experiencias sexuales .Una de las dimensiones relacionadas a los sentidos construidos en torno a las escolaridades que explicitan varias de las estudiantes entrevistadas, se anuda al diálogo que esas experiencias sostienen con las atravesadas por las generaciones familiares precedentes (Vasquez, 2013).En países como el Ecuador las expectativas de los adolescentes influyen notablemente en el objetivo 3 del plan nacional del buen vivir cuyo texto plantea mejorar la calidad de vida de la población (Senplades, 2013)

Para determinar si el personal de enfermería sabe aplica principios bioéticos en la calidad de atención se procedió a elaborar un test del nivel de satisfacción de los servicios brindados a las usuarias adolescentes en el área de ginecología del hospital básico de Jipijapa, así se logrará identificar los principales dilemas bioéticos que las enfermeras se ven obligadas a resolver en sus actividades diarias en el área de ginecología. 
Al realizar el estudio se pudo apreciar que el personal de enfermería del hospital básico Jipijapa conoce misión visión de los servicios que debe prestar, además de existir las buenas relaciones interpersonales entre el personal, sin embargo no manejan protocolos al presentarse problemas bioéticos pero existe el respeto al paciente y los familiares.

La Bioética es una disciplina que estudia la conducta humana, a la luz de los valores y principios morales tiene una injerencia muy importante en el cuidado que presta el profesional y sobre todo la parte humanística de Enfermería a las personas que acuden a los distintos centros de salud para solicitar la atención en este caso a las usuarias adolescentes. En tanto ética aplicada no logra refugiarse en convicciones personales, porque su campo de reflexión se centra en actividades interpersonales donde el pluralismo hoy vigente hace probable que la conciencia moral del agente enfrente valores diferentes reclamados por quienes requieren su intervención, los derechos humanos incluyen un cúmulo de varios aspectos sociológicos, antropológicos y políticos inclusive donde asuntos como el aborto por ejemplo tiene varios frente a favor y en contra. Tampoco es aceptable que una democracia bien constituida elabore políticas públicas cuyo tenor moral se sustenta en convicciones personales o grupales no compartidas por la sociedad civil. (Miguel, 2010)

Existe un deber general de respeto al paciente atendido por el personal de salud, que comprende el respeto por su autonomía e implica obtener su consentimiento para los tratamientos e intervenciones médicas; lo cual es válido también en los casos de menores de edad. Los métodos anticonceptivos que se usan actualmente en la adolescencia tienen unos riesgos mínimos para la salud y, en determinados casos, beneficios frente al riesgo que el embarazo representa para la salud física y psíquica. (Lamm, 2010). Sin embargo no está obligado el paciente a acceder a las indicaciones del personal de salud .Una de las situaciones más complejas en las que se puede encontrar el profesional de la salud es la que se produce cuando un paciente rechaza el tratamiento que se le propone. Ésta era una actuación impensable durante siglos, pero ahora se reconoce como uno de los derechos del paciente, si no el más básico, puesto que le otorga la capacidad decisoria sobre su integridad física (Azucena, 2012)

El principio bioético de justicia suele ser confuso en temas como el aborto .Mucho se debate hoy en día en torno a si resulta adecuado o no el exigir el consentimiento o autorización de los padres a las adolescentes que desean realizarse un aborto. Esta es una práctica que se exige prácticamente 
Rev SINAPSIS, Vol. 6, Nº 1, Junio 2015

en el 100\% de las naciones que tienen servicios de aborto despenalizado (Jorge, 2008). Al analizar las implicaciones que los cambios fisiológicos y psicológicos que tienen los adolescentes en esta etapa de su vida se percibe la necesidad de una adecuada orientación, para eso algunos estudios realizados han permitido determinar aspectos de la personalidad, investigadores como Parra comenzaron exponiendo las dos características esenciales que definen el pensamiento de los adolescentes: la impulsividad y una suerte de pensamiento mágico que les hace pensar que son invulnerables. Estos rasgos les llevan a desarrollar conductas de riesgo que pueden poner en peligro su salud. Por supuesto, los factores ambientales y contextuales influirán también de modo importante en esos rasgos y en las conductas de los adolescentes. (Lydia, 2013)

Según la Organización Panamericana de la Salud (OPS) la adolescencia es el período comprendido entre los 10 y 19 años, que incluye cambios biológicos, sicológicos y sociales. En cambio, el Código de la Niñez y Adolescencia del Ecuador considera que adolescentes la persona de ambos sexos entre 12 y 18 años. Para efectos legales de protección integral y especial se tomarán en cuenta estos dos conceptos. En los países de América latina donde aún encontramos deficientes políticas públicas que orienten la sexualidad de los adolescentes, no se ha delimitado claramente hasta qué punto tienen libertad de tomar desiciones.La anticoncepción protege la vida y la salud de las personas. Evita embarazos no deseados, abortos y permite establecer el momento adecuado para la concepción en mujeres con patologías que pueden alterar el embarazo. Un análisis pormenorizado de los aspectos éticos que pueden influir e incluso a veces mediatizar en las decisiones a adoptar en el terreno de la anticoncepción, nos obliga a considerar al menos algunos de los siguientes (Neyro-Bilbao J1, 2015)

El aborto, la anticoncepción, el segundo embarazo adolescente, la escolaridad deben de ser orientados para que el personal de salud sepa aplicar los principios Bioéticos en la calidad de atención integral a usuarias adolescentes atendidas en el área de ginecología del hospital investigado, para obtener datos se usó un test del nivel de satisfacción de los servicios brindados a las usuarias adolescentes en el área de ginecología del hospital básico de jipijapa Y así se logró identificar los principales dilemas bioéticos que las enfermeras se ven obligadas a enfrentar en sus actividades diarias en el área de ginecología además de identificarse la necesidad de que se elaboren protocolos en caso de suscitarse dilemas bioéticos que resolver.

El equipo de salud integrado por médicos y enfermeras debe estar preparado para afrontar los dilemas bioéticos que se presentan en la atención a usuarias adolescentes, ya que de acuerdo a la 
Rev SINAPSIS, Vol. 6, Nº 1, Junio 2015

calidad de atención recibida se podrá lograr que el bienestar no sólo sea percibido por la usuaria sino que también repercuta en los hijos de las adolescentes en el caso de que esté embarazada. Se concluye que los avances en la investigación y el rápido desarrollo de nuevas y costosas tecnologías acentúan los problemas éticos a los que se enfrentan los médicos, pero debemos aprender a equiparar ambos aspectos en la práctica cotidiana. Así, durante el embarazo, período de preocupación y ansiedad, las conversaciones eficaces con la mujer y su familia pueden contribuir a aumentar la confianza que ella deposite en el personal de salud, que se ven cada vez más arrastrados hacia diferentes direcciones; por un lado, los intereses de pacientes individuales y, por otro, el compromiso con la sociedad y las generaciones no nacidas todavía (Valdés Yong Magel, 2014)

La enfermera debe empoderar al adolescente para que asuma su dignidad y su valor como persona quién por tanto es el constructor de su propia historia. Debe ayudarle a reconocer el valor de su vida y de su cuerpo encontrándole propósito a la vida. Una adolescente correctamente orientada en aspectos que incluyen la aplicación de principios bioéticos será quien lleve adelante a un país o pase a formar parte de los cinturones de pobreza, claro está que no sólo depende del personal de salud sino también de otros actores, pero cada uno de ellos haciendo en su lugar de trabajo las actividades de la mejor manera posible se podrá orientar a una generación que está en constante evolución.

De tal manera que se aprecian nuevas configuraciones en materia de atención a adolescentes. La adolescencia sino participación interactiva permanentemente con ellos (Mirtha, 2002)

\section{Metodología}

Para llevar a efecto el desarrollo de esta investigación se realizarón a través del método inductivo, descriptivo y analítico. Con la finalidad de determinar una muestra estadística representativa de la población de usuarias adolescentes que reciben atención en el área de ginecología aplicamos la respectiva formula, tomando en cuenta la totalidad de la población (144 usuarias adolescentes ingresadas desde mayo a octubre del 2013) mientras que la totalidad del personal de enfermeras (15) Por ser una muestra pequeña serán considerados toda la población en la presente investigación. 
Rev SINAPSIS, Vol. 6, Nº 1, Junio 2015

Criterios de inclusión: Usuarias adolescentes hospitalizadas, edad entre 13 y 19 años, personal de enfermería, autónomos y en pleno uso de sus facultades mentales.

Criterios de exclusión: Usuarias adolescentes que no hayan estado embarazadas y personal médico que laboraba en el área de ginecología.

Las técnicas que se utilizó en esta investigación fue:

- Reconocimiento del área

- Observación

- Cuestionario de encuestas

\section{Resultados}

Al consultarles a las usuarias los motivos por los que regresan al centro de salud sus respuestas fueron: Principalmente la amabilidad y el respeto con las que son atendidas luego la forma profesional con la que actúan las enfermeras y la capacitación que de ellas reciben las usuarias

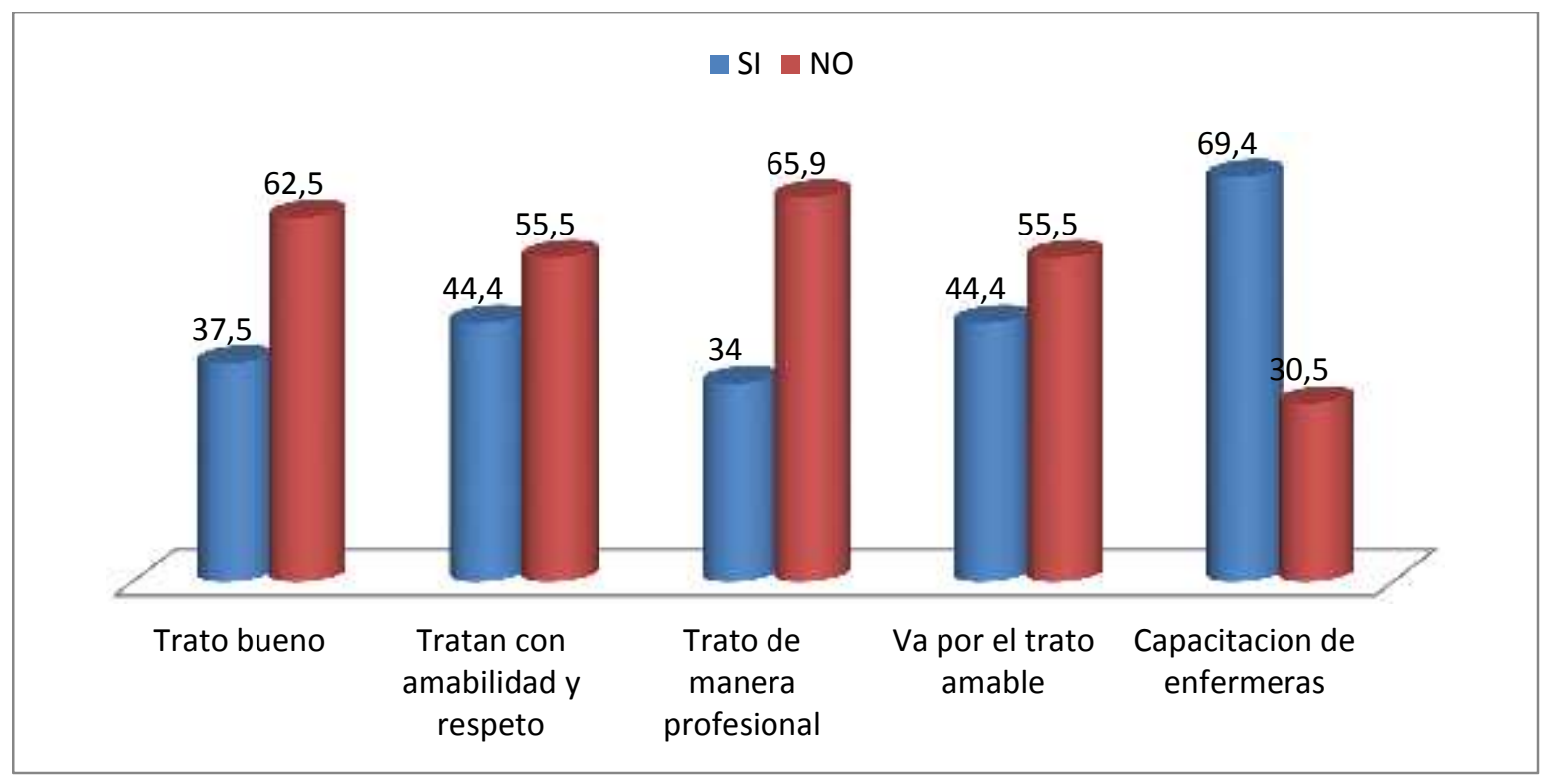

En el test aplicado a las usuarias de ginecología de un total de 144, 90 adolescentes correspondiendo al $62,5 \%$ mencionaron que el trato que le brinda el personal de enfermería no es bueno; sin embargo 54 usuarias optaron por la opción si es bueno el trato que le brindan el personal de enfermería, además de identificarse en las respuestas los motivos por los que ellas consideran el buen trato o no. 
Rev SINAPSIS, Vol. 6, Nº 1, Junio 2015

Las enfermeras fueron interrogadas para determinar si manejan protocolos al presentarse dilemas bioéticos, dando a conocer las siguientes respuestas

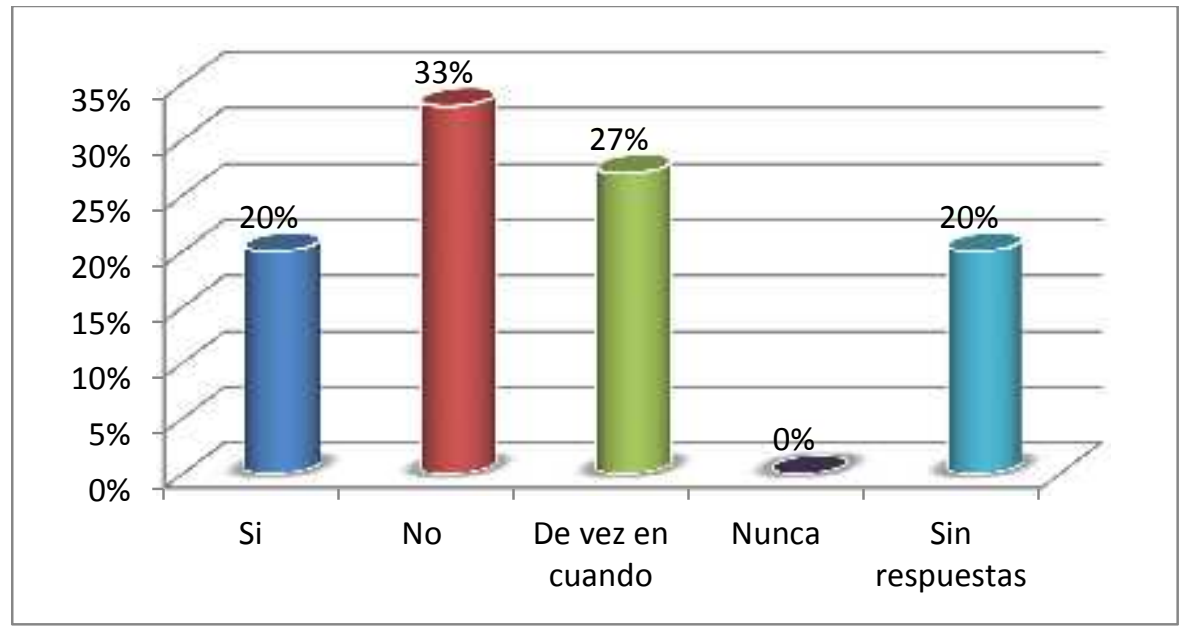

Las enfermeras respondieron que la aplicación de protocolos en el caso de dilemas bioéticos ocurre en un $20 \%$, no se aplican en un 33\%, y el restante dijo no aplicarlos o no respondió

\section{Al interrogar a las enfermeras sobre si prioriza y coordina el tiempo dedicado a cada paciente, sus respuestas fueron:}

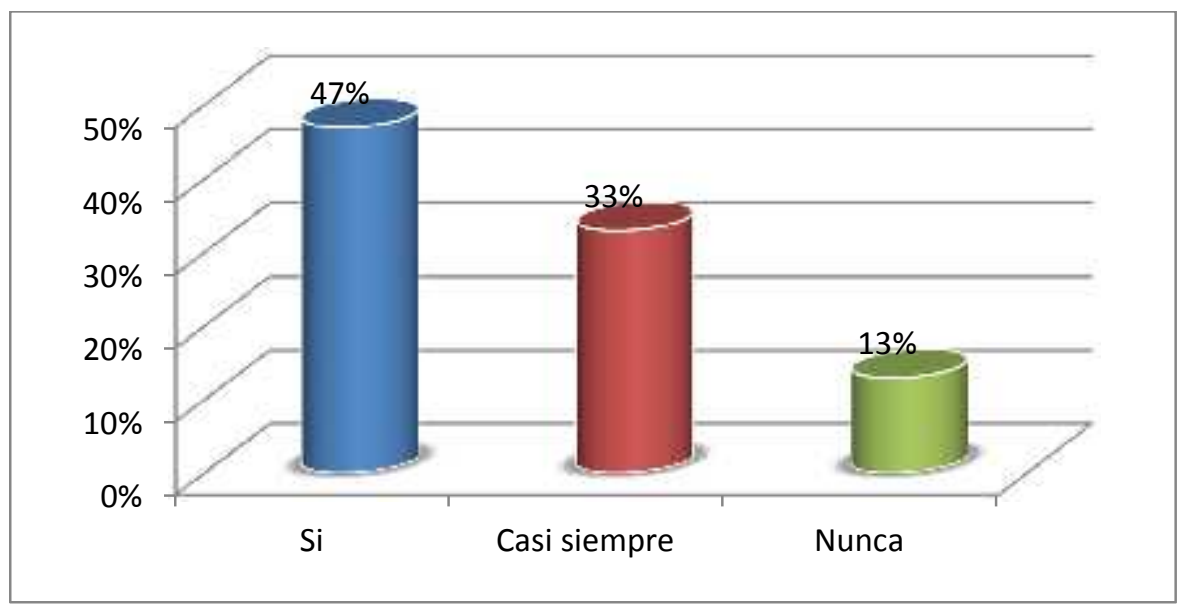

Percepción de las adolescentes respecto a si cubre o no sus necesidades inmediatas el personal de enfermería 
Rev SINAPSIS, Vol. 6, No 1, Junio 2015

ISSN $1390-7832$

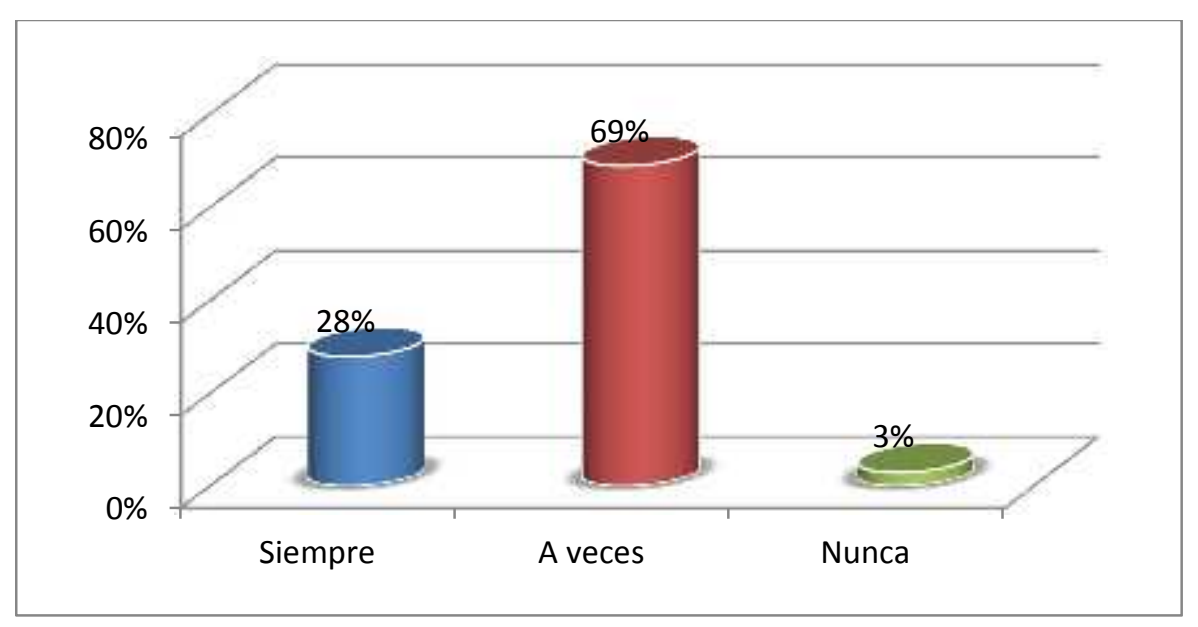

\section{Discusión}

El embarazo en la adolescencia es una preocupación no solo de estos tiempos. Estudios realizados han demostrado un rejuvenecimiento en la fecundidad con el objetivo de evaluar la repercusión biopsicosocial que tiene en este grupo etáreo, por tanto es una inquietud por los riesgos que traen consigo no solo a la salud, sino también en la vida social de las adolescentes, sin embargo una vez embarazadas es de vital importancia darle el tratamiento que necesitan desde el punto de vista médico y más aún por el personal de enfermería que brinda cuidados directos y acompaña a los pacientes mucho más tiempo. En la adolescencia hay cambios biopsicosociales y principalmente se afecta la esfera afectiva, a estos cambios se añaden los que fisiológicamente ocurren en el embarazo que también tienen gran repercusión en la esfera emocional, por lo tanto es necesario aplicar todos los conocimientos relacionados con la Psicología, con la Medicina, con la Ginecología y mucho más importantes con la Bioética para evitar complicaciones relacionadas con estrés emocional que pueden desencadenar partos prematuros y poner en peligro la vida tanto de la madre como del niño que está por nacer.

La enfermera/o en su desempeño profesional debe brindar cuidados en correspondencia con las necesidades de salud de las personas, las familias y las comunidades en su ambiente social y natural, pero además ha de considerar lo que se derive del nivel de desarrollo actual y perspectivo del contexto y sus exigencias, aunque no siempre las decisiones en algunos ámbitos de salud y en este caso los que tienen que ver con la planificación familiar sean los más acertados. 
Rev SINAPSIS, Vol. 6, Nº 1, Junio 2015

ISSN $1390-7832$

La maternidad es una prioridad en la atención de enfermería en cualquier grupo etario y corresponde al personal de la salud asumir este reto y minimizar las complicaciones que puedan derivarse del embarazo.

S.J. Alfonso Llano Escobar en una revista de la especialidad, define a la Bioética como "el uso creativo del diálogo inter y transdisciplinaria entre ciencias de la vida y valores humanos. Sin embargo, cabe destacar, que ya en 1978, el Kennedy Institute de la Universidad jesuita de Georgetown en Estados Unidos, había publicado la primera Enciclopedia de Bioética en cuatro volúmenes, dirigida por Warren Reich, un teólogo católico, donde se define a la Bioética como el "estudio sistemático de la conducta humana en el área de las ciencias de la vida y la salud, examinado a la luz de los valores y principios morales".

Dentro de los principios de la Bioética está el relacionado con la beneficencia que consiste en la obligación de actuar en beneficio de otros, promoviendo sus legítimos intereses y suprimiendo prejuicios, así como el de no maleficencia que se refiere a abstenerse intencionadamente de realizar acciones que puedan causar daño o perjudicar a otros. En medicina, sin embargo, este principio debe encontrar una interpretación adecuada pues a veces las actuaciones médicas dañan innecesariamente a otros.

Partiendo de la base que la Enfermería como profesión constituye un servicio encaminado a satisfacer las necesidades de salud de las personas sanas o enfermas, individual o colectivamente, y teniendo en cuenta los factores que influyen en las profesiones sanitarias como es la interculturalidad, el movimiento mundial sobre el desarrollo sostenible, la cultura por la paz y el rechazo a la violencia en todas sus formas, no es digno ni legalmente permitido, el maltrato en cualquiera de sus manifestaciones en el ejercicio de la profesión (OMS)

Por tanto vale destacar que el embarazo en la adolescencia es un problema de salud en el ámbito internacional y por ende su atención esta priorizada en cuanto a reglas, normas o protocolos de atención que rigen a las instituciones de salud públicas o privadas, siendo la enfermera por el rol que desempeñan en la sociedad protagonistas en el cumplimento legal de estas normas.

La enfermera tiene el compromiso moral y social de cuidar con calidad humana, científica, técnica y ética a las personas sanas /enfermeras. Compromiso social exige a la enfermera capacitación, 
Rev SINAPSIS, Vol. 6, Nº 1, Junio 2015

ISSN 1390 - 7832

sensibilidad ética, humanismo y capacidad de razonamiento moral para la adecuada toma de decisiones éticas ante dilemas éticos en su práctica profesional (Canto 2008)

La bioética es una potente herramienta moral y legal, cuyos objetivos se dirigen hacia el bien del individuo y en aras de la conservación de la salud y de la vida, toma y considera al ser humano en su estrecha relación con los factores ambientales como naturaleza, cultura, religión, política y sociedad, entre otros, respetando la individualidad de cada ser y la pluralidad de escenarios posibles (García 2013)

Estos estudios demuestran que el embarazo en la adolescencia es un problema de salud en el ámbito internacional y por ende su atención esta priorizada en cuanto a reglas, normas o protocolos de atención que rigen a las instituciones de salud públicas o privadas, siendo la enfermera por el rol que desempeñan en la sociedad protagonistas en el cumplimento legal de estas normas, por lo tanto es necesario que briden servicios de excelencia en cuanto a atención (Canto. M, 2013)

\section{Conclusiones}

El personal de enfermería del hospital básico Jipijapa conoce misión visión de los servicios que debe prestar, además de existir las buenas relaciones interpersonales entre el personal, sin embargo no manejan protocolos al presentarse problemas bioéticos pero existe el respeto al paciente y los familiares.

Las adolescentes consideran que las enfermeras en algunos casos no le brindan un trato bueno, ni humanístico y deben tener capacitaciones para mejorar el trato con sus pacientes además consideran que a veces las enfermeras prestan calidad de atención, rapidez y profesionalismo, a su vez expresaron que siempre les brindan la información sin embargo solo a veces satisfacen las necesidades de los pacientes

Mediante la observación directa se pudo conocer los diferentes dilemas bioéticos que se ven obligadas a enfrentar en sus actividades las enfermeras en el área de ginecología, se percibe la necesidad de que el personal de enfermería tenga capacitación continua sobre bioética

\section{Bibliografía}


Rev SINAPSIS, Vol. 6, № 1, Junio 2015

ISSN $1390-7832$

1. Azucena, C. (2012). La enseñanza práctica de la bioética :Objetivos, diseño curricular y materiales docentes. Revista educación en ciencias de la salud, 69-80.

2. Canto. M, G. L. (2013). La bioética y los derechos de las adolescentes en el aborto. Revista Cubana de Obstetricia y Ginecología, 39(4), 56.

3. Ecuador, M. d. (2012). Estrategia Nacional Intersectorial de Planificación Familiar y Prevención del Embarazo Adolescente. Quito-Ecuador: Ministerio de Salud Pública .

4. Garcia, s. m. (2013). aprobada la declaración universal sobre bioética y derechos humanos de la unesco. Unesco, 12.

5. Ginecología, F. L. (2013). factores relacionados con el embarazo y la maternidad en menores de 15 años en américa latina y el caribe. En c. d. reproductivos, factores relacionados con el embarazo y la maternidad en menores de 15 años en américa latina y el caribe (pág. 23). buenos aires: federacion latinoamericana de sociedades de obstetricia y ginecología.

6. joaquín, O. (Volumen 70, No. 1, enero-febrero 2002). la Bioética y su relación médico-paciente. cirujía y cirujanos, 55-59.

7. Jorge, P. M. (2008). Aborto en la adolescencia .Consideraciones bioéticas en torno a la desición de abortar. Revista cubana de Obstetricia y Ginecología, 34.

8. Lamm, M. C. (2010). inofrme del observatorio de bioetica. Barcelona: Ind Can Calderón.

9. Lydia, F. (2013). El menor maduro, XVI Ateneo de Bioética. Revista de la Fundación Ciencias de la Salud, 4.

10. Miguel, K. (2010). Objeción de conciencia bioética. Chile: Cuad Med Soc Chile.

11. Mirtha, S. C. (2002). Bioética, Complejidad y adolescencia. Bogotá: Universidad del Bosque.

12. Neyro-Bilbao Jl, E. M. (2015). Anticoncepción y bioética: entre la objeción de conciencia y el prinncipio de autonomía. Ginecología Obstetricia México, 128.

13. Senplades. (2013). Plan Nacional de Buen vivir. Quito: Secretaria nacional de planificacion ecuador.\}

14. Valdés Yong Magel, H. N. (2014). La bioética en el desarrollo de la ginecobstetricia. Revista Cuabana de Investigaciones Biomédicas, 433. Obtenido de http://scielo.sld.cu

15. Vasquez, L. M. (2013). alumnas embarazadas y/o madres. pruebas escolares, soportes y resistencias en contextos de marginalidad urbana. facultad latinoamericana de ciencias sociales, sede argentina, maestría en ciencias sociales con orientación en educación, 13-23. 